Franck DELVARE · Alain CIMETIERE.

\title{
A first order method for the Cauchy problem for the Laplace equation using BEM
}

Received: date / Accepted: date

\begin{abstract}
The purpose is to propose an improved method for inverse boundary value problems. This method is presented on a model problem. It introduces a higher order problem. BEM numerical simulations highlight the efficiency, the improved accuracy, the robustness to noisy data of this new approach, as well as its ability to deblur noisy data.
\end{abstract}

Keywords Cauchy problem · inverse problem · boundary values $\cdot$ boundary element method $\cdot$ regularization

\section{Introduction}

The purpose is to propose an improved method using boundary element method (BEM) to solve Cauchy inverse problems. These problems are called data completion problems. They consist in recovering data on a part of the boundary given the partial differential equation within the domain and overspecified data available on the remaining part of the boundary. This kind of problems arises in many engineering fields like thermal problems, electrostatic or elastostatic ones.

For convenience, we will present the new method in the framework of a model problem: the Cauchy problem for the Laplace equation. Other methods exist to solve this problem. The main methods are Tikhonov like methods, [8], [11], [17], [18], quasi reversibility methods [3], [9], [13], [15], and iterative methods [1], [12], [14], [16]. Quasi reversibility methods and Tikhonov regularization methods present the

Franck DELVARE

Laboratoire Energétique Explosions Structures

Université d'Orléans and ENSI de Bourges

88 Boulevard Lahitolle, 18020 Bourges Cedex, France

E-mail: franck.delvare@ensi-bourges.fr

Alain CIMETIERE

LMP, Université de Poitiers

Boulevard Marie et Pierre CURIE,Téléport 2

BP 30179, 86962 Futuroscope Chasseneuil Cedex, France avantage to lead to well-posed problems after modifying the partial derivative operator. The iterative methods, where the number of iterations acts as a regularization parameter, are based on the use of a sequence of well-posed problems.

In previous works [4],[6] we have presented a somewhat different resolution approach for the Cauchy problem for the Laplace equation. This approach was introduced in [4] and reduces to the resolution of a sequence of optimization problems under equality constraints. The functional is composed of two terms. The first one represents the gap between the optimal element and the overspecified data, the second one the gap between the optimal element and the previous optimal element (regularization term). The equality constraints characterize the equilibrium. So, we obtain at each step an optimal element which is an exact equilibrium and is near to the overprescribed data. In case of compatible data, we have also proved that the sequence limit is the solution of the Cauchy problem.

This method was numerically implemented using both the finite element method [4] and the boundary element method [6]. We obtained the same results in both situations, accuracy and robutness features especially for the function.

In order to improve the reconstruction of the normal derivatives, reduce the extension of the boundary part where overspecified data are given and deblur noisy normal derivatives data, we have introduced [5] a higher order method which will be called first order method.

This paper is devoted to the numerical implementation of the first order method using BEM. The efficiency of the proposed method, as well as its robustness and ability to deblur noisy data will be highlighted.

\section{The first order method}

2.1 The Cauchy problem for the Laplace equation

The presentation of the method is carried out for the model problem (1), where $\Omega$ is a two-dimensional bounded domain and $\Delta$ denotes the Laplacian operator. On the part $\Gamma_{d}$ of the boundary $\Gamma=\partial \Omega$, overspecified boundary conditions are 
given. The aim is to calculate $u$ and its normal derivative denoted by $u^{\prime}$ on $\Gamma_{u}=\Gamma \backslash \Gamma_{d}$.

$$
\left\{\begin{array}{l}
\Delta u=0 \text { in } \Omega \\
u=\phi_{d} \text { on } \Gamma_{d} \\
u^{\prime}=\psi_{d} \text { on } \Gamma_{d}
\end{array}\right.
$$

The problem (1) admits a unique solution when the data $\phi_{d}$ and $\psi_{d}$ are compatible, but its solution is very sensitive to small data perturbations, since Cauchy inverse problems are ill-posed problems [10].

\subsection{The first order problem}

In order to improve the reconstruction of $u^{\prime}$, we introduce the following first order method:

$$
\left\{\begin{array}{l}
\Delta u=0, \quad \Delta u_{1}=0, \quad \Delta u_{2}=0 \text { in } \Omega \\
u^{\prime}=u_{1} n_{1}+u_{2} n_{2} \text { on } \Gamma \\
\int_{\Gamma}\left(u_{1} n_{1}+u_{2} n_{2}\right) d s=0 \\
u=\phi_{d}, \quad u_{1}=\phi_{1 d}, \quad u_{2}=\phi_{2 d}
\end{array}\right.
$$

where $n_{1}$ and $n_{2}$ are the outward normal components.

The first order method is associated with the problem (2). The expressions of the additional data $\left(\phi_{1 d}, \phi_{2 d}, \psi_{1 d}\right.$ and $\left.\psi_{2 d}\right)$ are related to $\phi_{d}$ and $\psi_{d}$ by (3):

$$
\begin{cases}\phi_{1 d}=\psi_{d} n_{1}-\frac{d \phi_{d}}{d s} n_{2}, & \phi_{2 d}=\psi_{d} n_{2}+\frac{d \phi_{d}}{d s} n_{1} \\ \psi_{1 d}=-\frac{d \phi_{2 d}}{d s}, & \psi_{2 d}=\frac{d \phi_{1 d}}{d s}\end{cases}
$$

When the data $\phi_{d}$ and $\psi_{d}$ are compatible and the solution $u$ is sufficiently regular, all the data of the problem (2) are also compatible. Then $u$ is the solution of the problem (1) and $u_{1}$ and $u_{2}$ are respectively the two partial derivatives of $u$.

\subsection{Equivalent formulation for the first order problem}

We rewrite the problem (2) distinguishing the equilibrium equations (4)

$$
\left\{\begin{array}{l}
\Delta u=0, \Delta u_{1}=0, \quad \Delta u_{2}=0 \text { in } \Omega \\
\int_{\Gamma}\left(u_{1} n_{1}+u_{2} n_{2}\right) d s=0
\end{array}\right.
$$

and the boundary conditions (5)

$\begin{cases}u=\phi_{d}, \quad u_{1}=\phi_{1 d}, \quad u_{2}=\phi_{2 d} & \text { on } \Gamma_{d} \\ u^{\prime}=\psi_{d}, \quad u_{1}^{\prime}=\psi_{1 d}, \quad u_{2}^{\prime}=\psi_{2 d} & \text { on } \Gamma_{d} \\ u^{\prime}=u_{1} n_{1}+u_{2} n_{2} \text { on } \Gamma & \end{cases}$

Given $\Phi_{d}=\left(\phi_{d}, \phi_{1 d}, \phi_{2 d}, \psi_{d}, \psi_{1 d}, \psi_{2 d}\right)$, an equivalent formulation for (2) is given by (6), where $H(\Gamma)$ is a space of traces of functions satisfying (4).

$$
\left\{\begin{array}{l}
\text { Find } U=\left(u, u_{1}, u_{2}, u^{\prime}, u_{1}^{\prime}, u_{2}^{\prime}\right) \in H(\Gamma) \text { such that : } \\
U=\Phi_{d} \text { on } \Gamma_{d} \\
u^{\prime}=u_{1} n_{1}+u_{2} n_{2} \text { on } \Gamma
\end{array}\right.
$$

The problem (2) and its reformulation (6) are ill-posed problems in Hadamard's sense.

\subsection{The iterative regularizing algorithm}

In order to solve the ill-posed problem (6), we introduce the iterative regularizing algorithm (7):

$$
\left\{\begin{array}{l}
\text { Let us consider } c>0 \text { and } U^{0} \in H(\Gamma) \\
\text { Find } U^{k+1} \in H(\Gamma) \text { such that }: \\
J_{c}^{k}\left(U^{k+1}\right) \leq J_{c}^{k}(V) \forall V \in H(\Gamma) \text { with } \\
J_{c}^{k}(V)=\left\|v^{\prime}-\left(v_{1} n_{1}+v_{2} n_{2}\right)\right\|_{-\frac{1}{2}, \Gamma}^{2} \\
+\left\|V-\Phi_{d}\right\|_{\Gamma_{d}}^{2}+c\left\|V-U^{k}\right\|_{\Gamma}^{2}
\end{array}\right.
$$

It is based on the same idea as the algorithm proposed in [6]. The ill-posed problem (2) is replaced by a sequence of wellposed optimization problems in $H(\Gamma)$. In order to remove instabilities, the last term is introduced. It is a control term on the whole boundary $\Gamma$. The optimal elements obtained at each step are solutions of the equilibrium equations (4).

When the data $\left(\phi_{d}, \psi_{d}\right)$ are compatible, it is also proved that this iterative process converges for all $U^{0}$ in $H(\Gamma)$ and for all $c>0$. The first component of the limit is associated with the solution of problem (1). The proof is similar to the one carried out in [6].

\section{BEM implementation and numerical simulations}

\subsection{BEM discretization of the iterative algorithm}

\subsubsection{The boundary element method}

The boundary element method [2] is used to discretize the iterative algorithm (7) and specially to obtain a discrete representation of the $H(\Gamma)$ space.

By using the usual fundamental solution of the Laplace equation and Green's identities then the governing partial differential equation $\Delta u=0$ is transformed into the following integral equation [2]:

$\eta(x) u(x)=\int_{\Gamma}\left(G(x, y) \frac{\partial u(y)}{\partial n}-u(y) \frac{\partial G(x, y)}{\partial n}\right) d \Gamma_{y}$

where $\eta(x)=1$ if $x \in \Omega$ and $\eta(x)=\frac{1}{2}$ if $x \in \Gamma$ (smooth), and $G$ is the fundamental solution of the Laplace equation which in two dimensions is given by

$G(x, y)=-\frac{1}{2 \pi} \ln (r), \quad r^{2}=\left(x_{1}-y_{1}\right)^{2}+\left(x_{2}-y_{2}\right)^{2}$

In practice the boundary integral equation cannot be solved analytically and thus some form of numerical approximation is needed. Generically, if the boundary $\Gamma$ is discretized into $N$ boundary elements and the boundary integral equation is applied at the midpoint of each element, then a system of $N$ linear algebraic equations is obtained in the form

$A U+B U^{\prime}=0$

where $A$ and $B$ are matrices which only depend on the geometry of the boundary and the vectors $U$ and $U^{\prime}$ are respectively the discretized values of the function and its normal derivative, which are assumed to be constant over each 
boundary element and take their values at the midpoint of each element.

Expression (10) represents a system of $N$ linear algebraic equations with $2 N$ unknowns.

\subsubsection{Discrete representation of the space $H(\Gamma)$}

The boundary element method leads to the definition of $H_{h}(\Gamma)$, the $H(\Gamma)$ discretization. $H_{h}(\Gamma)$ reads as follows:

$H_{h}(\Gamma)=\left\{\begin{array}{l}\Psi=\left(U, U_{1}, U_{2}, U^{\prime}, U_{1}^{\prime}, U_{2}^{\prime}\right) \in R^{6 N}: \\ A U+B U^{\prime}=0 \\ A U_{1}+B U_{1}^{\prime}=0 \\ A U_{2}+B U_{2}^{\prime}=0 \\ N_{1}^{T} U_{1}+N_{2}^{T} U_{2}=0\end{array}\right\}$

where $U_{1}, U_{2}, U_{1}^{\prime}$ and $U_{2}^{\prime}$ are respectively the discretized values of $u_{1}$ and $u_{2}$ and their normal derivatives and where $N_{1}, N_{2}$ are the discretized values of the unit normal components over each boundary element.

Expression (11) represents a system of $3 N+1$ linear algebraic equations with $6 N$ unknowns.

\subsubsection{Discretization of the iterative algorithm}

In the discrete case, the equivalence of the norms in finite dimension makes it possible to replace the $H^{-\frac{1}{2}}(\Gamma)$ norm by the $L^{2}(\Gamma)$ norm. We also take in all situations $U_{0}=0$ as initialization. The parameter $c$ value influences only the convergence rate. For non-noisy data, one takes a value $c=10^{-4}$ and for noisy data, a value $c=1$. In all cases, the algorithm converges in less than 100 iterations.

\section{Description of one step}

A discrete formulation of the continuous iterative process (7) reads as follows:

$$
\left\{\begin{array}{l}
\text { Find } \Psi^{k+1} \in H_{h}(\Gamma) \text { such that : } \\
J_{h c}^{k}\left(\Psi^{k+1}\right) \leq J_{h c}^{k}(\Psi) \quad \forall \Psi \in H_{h}(\Gamma)
\end{array}\right.
$$

This optimization problem is equivalent to the following optimization process under equality constraints:

$$
\left\{\begin{array}{l}
\text { Find } \Psi^{k+1} \in R^{6 N} \text { such that }: \\
J_{h c}^{k}\left(\Psi^{k+1}\right) \leq J_{h c}^{k}(\Psi) \quad \forall \Psi \in R^{6 N} \\
\text { under the equality constraints }: \\
A U+B U^{\prime}=0 \\
A U_{1}+B U_{1}^{\prime}=0 \\
A U_{2}+B U_{2}^{\prime}=0 \\
N_{1}^{T} U_{1}+N_{2}^{T} U_{2}=0
\end{array}\right.
$$

We introduce a $3 N+1$ vector of Lagrange multipliers to take into account the $3 N+1$ equality constraints. Each iteration in the algorithm needs to solve a system of $9 N+1$ linear algebraic equations with $9 N+1$ unknowns.
Some comments and remarks

The obtained matrix is symmetric. It is the same for each step of the iterative process and so needs to be computed and decomposed by a Crout factorization only once. From the second step of the algorithm, only actualization of the second member is needed.

The computational time for the first step of the algorithm (computation of different matrices, Crout decomposition) is , equivalent to the time needed for three hundred additional iterations.

The stopping test is based on a comparison between two successive optimal elements.

\subsection{Evaluation of additional data}

The evaluation of additional data by the use of the relations (3) needs differentiations of the data $\phi_{d}$ and $\psi_{d}$. First it is proceeded to an interpolation by B-splines of these data [7]. Then the differentiations are obtained by the analytical differentiation of the B-splines. For further details, the reader can see the description of the computational algorithm (figure 1). This is a way to obtain additional data but we have already used quadratic regression to compute differentiations and obtained similar results [5].

The input data for the inversion algorithm are on one hand the data $\phi_{d}$ and $\psi_{d}$ and on the other hand the generated additional data $\phi_{1 d}, \phi_{2 d}, \psi_{1 d}$ and $\psi_{2 d}$. It can be noticed that the interpolated functions $\phi_{d}$ and $\psi_{d}$ of the data are only used to evaluate differentiations of the given data.

\subsection{Numerical simulations}

This section is devoted to compare numerical results obtained by the proposed method (first order method) to whose obtained by the inverse method introduced (so called previous method) in [6].

The function $u$ to be recovered is defined by:

$$
u(x, y)=\cos (x) \cosh (y)+\sin (x) \sinh (y) .
$$

\subsubsection{Smooth boundary}

In this section, numerical simulations are performed on the domain $\Omega$ which is the disk with radius $\frac{1}{2}$ centered at point $\left(\frac{1}{2}, \frac{1}{2}\right)$. In the whole section, the boundary mesh is a regular one.

Behaviour with respect to boundary mesh

In this section, half of the boundary carries prescribed data. Figure 2 (respectively Figure 3 ) displays $L^{2}$ errors on $u$ (respectively on its normal derivative $u^{\prime}$ ) obtained by our previous method [6] and by the first order method, with respect to the number of boundary elements. We can notice that the first order method is stable with respect to boundary mesh as the previous method. But the first order method 


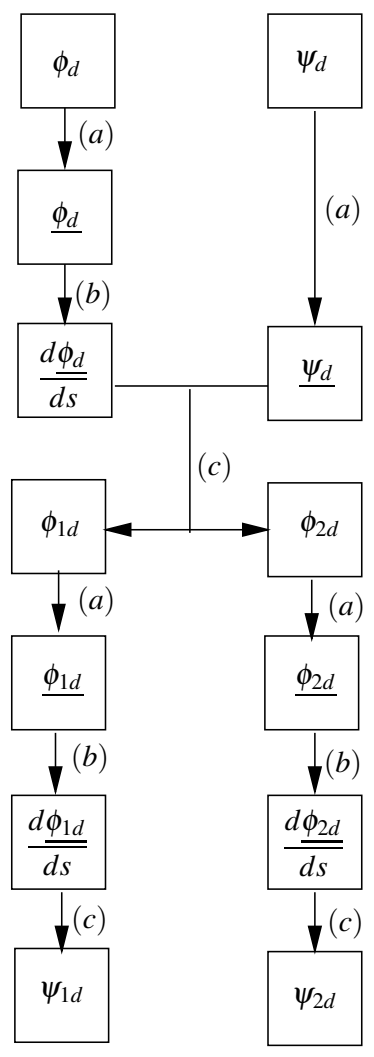

(a) B-Splines interpolation

(b) Analytic differentiation

(c) Use of relations (3)

Fig. 1 Computational algorithm for additionnal data evaluations

is more accurate. Errors obtained on function (respectively normal derivative) reconstructions are roughly five (respectively ten) times less.

Behaviour with respect to $\Gamma_{d}$ boundary extension

In this section, we introduce the ratio $\rho$ between the measures of $\Gamma_{d}$ and $\Gamma$. The number of boundary elements is fixed to 180 . Figure 4 (respectively Figure 5) displays $L^{2}$ errors on $u$ (respectively on its normal derivative $u^{\prime}$ ) obtained by our previous method [6] and by the first order method with respect to the ratio $\rho$. We can notice that the first order method is again more accurate than the previous method. As a consequence, the $\Gamma_{d}$ extension can be reduced by the use of the first order method.

Figure 6 (respectively Figure 7) gives the function (respectively its normal derivative) reconstructions for $\rho=\frac{1}{4}$ using the previous method and the first order one.

We can notice that the normal derivative reconstruction (Figure 7) is improved by the first order method. This reconstruction is as accurate as the function reconstruction (Figure 6) obtained by the previous method.

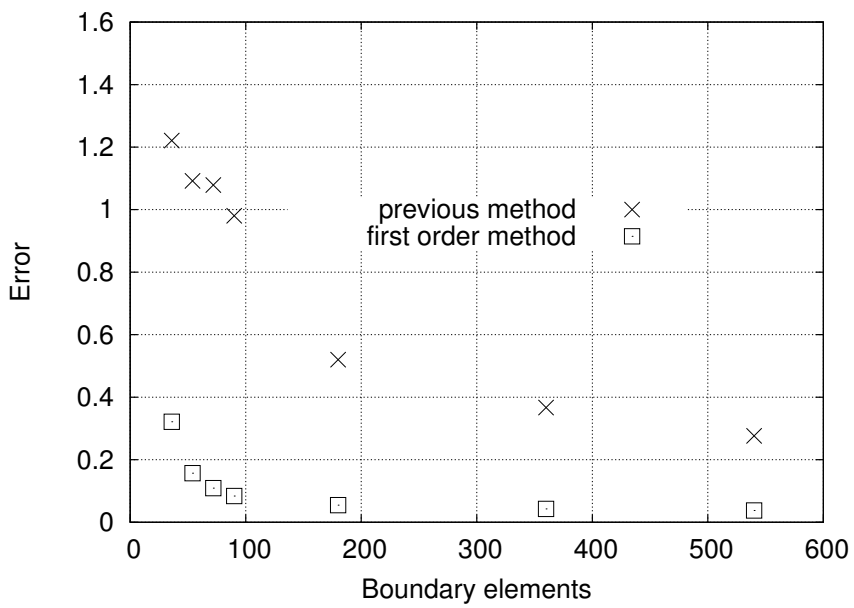

Fig. 2 Error $L^{2}(\Gamma)$ (in \%) on the function with respect to the boundary mesh

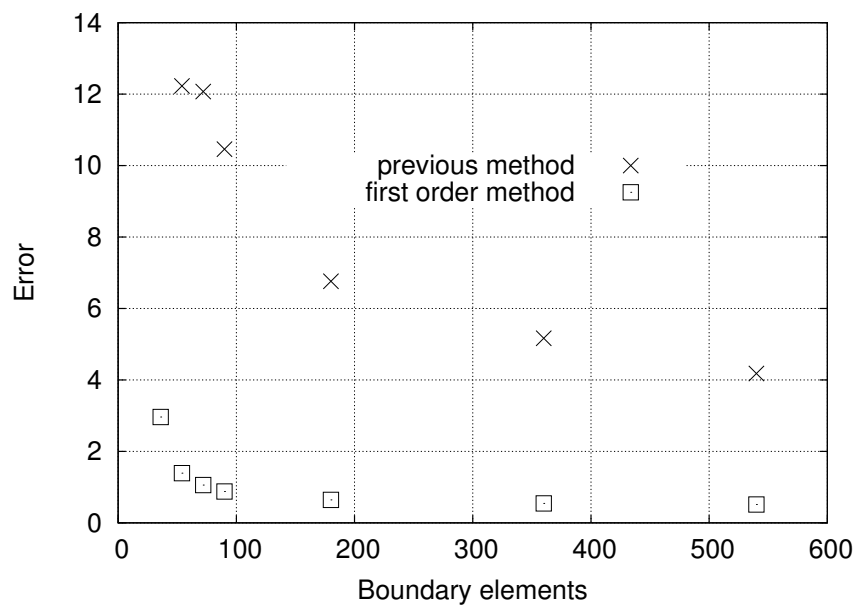

Fig. 3 Error $L^{2}(\Gamma)$ (in \%) on the normal derivative with respect to the boundary mesh

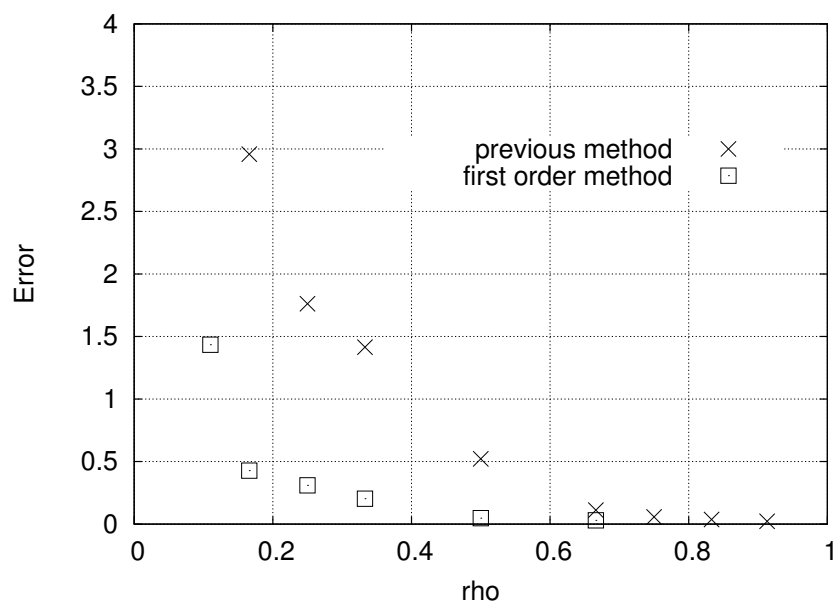

Fig. 4 Error $L^{2}(\Gamma)$ (in \%) on the function with respect to the ratio $\rho$ of given data 


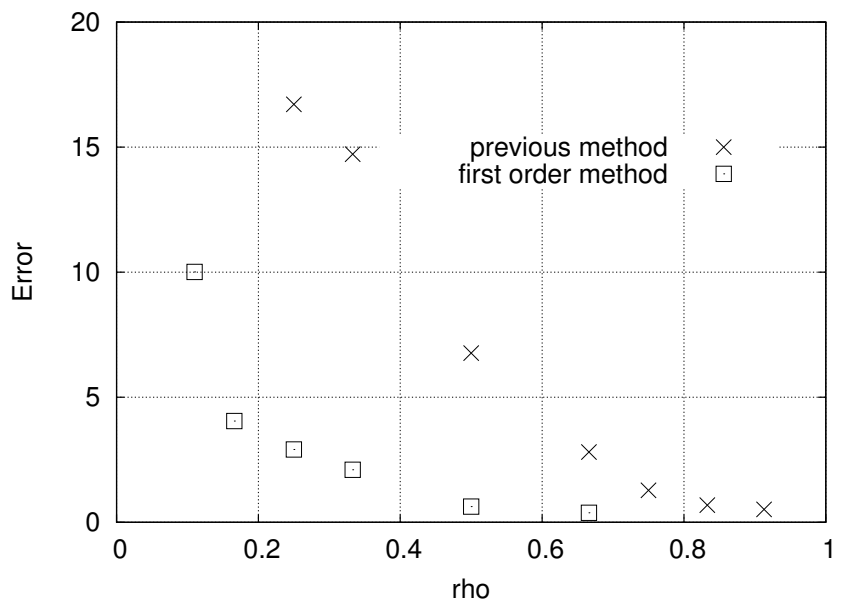

Fig. 5 Error $L^{2}(\Gamma)$ (in \%) on the normal derivative with respect to ratio $\rho$ of given data

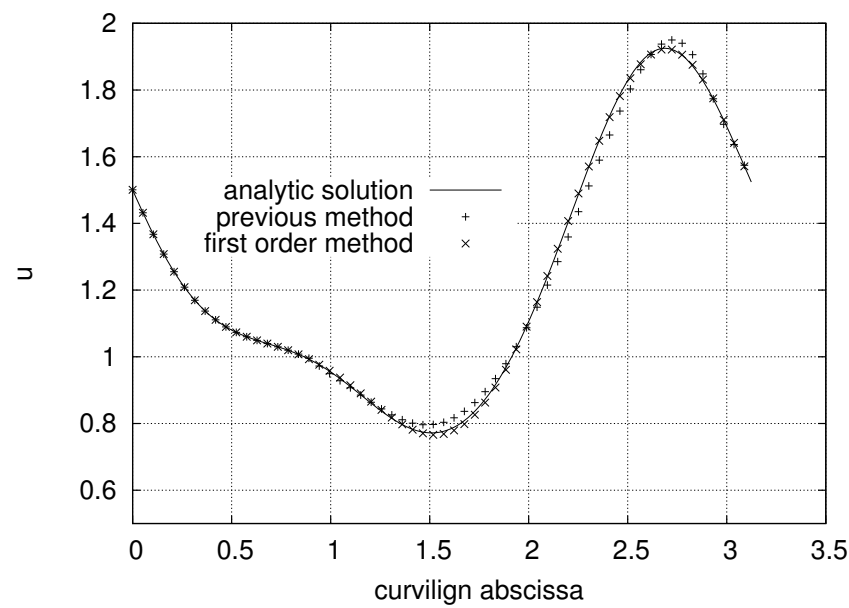

Fig. 6 Reconstructions of the function for $\rho=\frac{1}{4}$

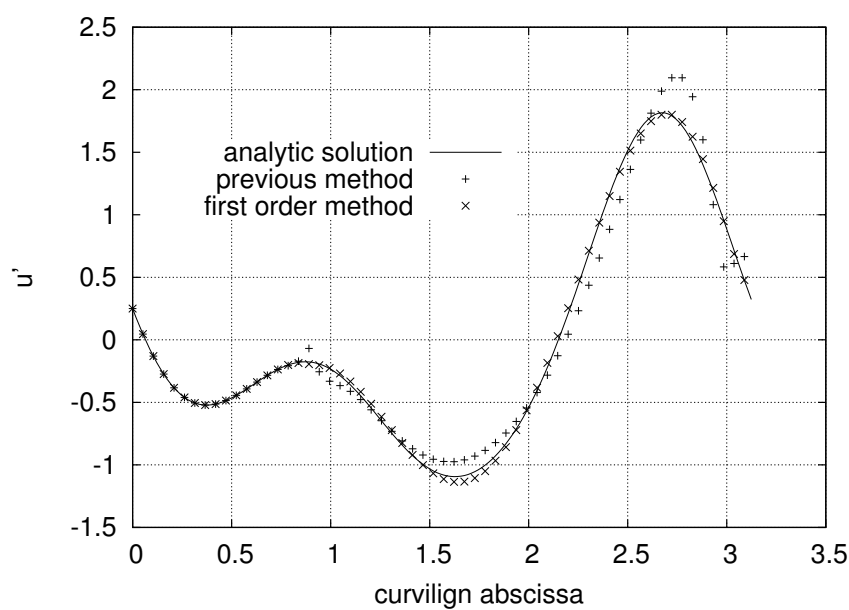

Fig. 7 Reconstructions of the normal derivative for $\rho=\frac{1}{4}$

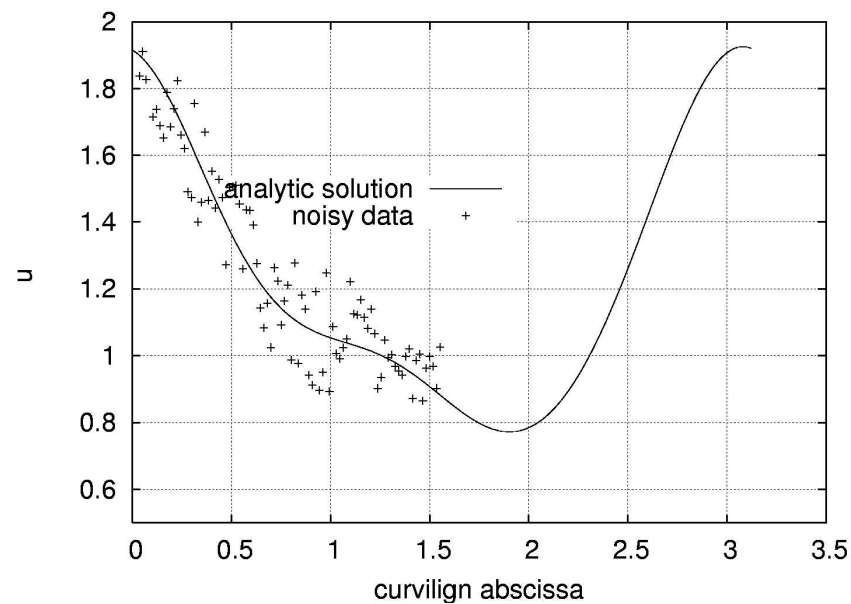

Fig. $8 \phi_{d}$ noisy data (10\%)

Behaviour with respect to noisy data

In this section, we discuss the numerical stability of the algorithm to noisy data. Half of the boundary carries prescribed data, the mesh is a 180 elements regular one. For each case, the noise is a random one whose amplitude is equal to $10 \%$ of the data maximum value on the boundary.

Firstly, we look at the algorithm stability when the data $\phi_{d}$ is noisy. Figure 8 gives the noisy data used by the algorithm. Figures 9 and 10 give the function and its normal derivative reconstruction.

We can notice that the algorithm by recomputing informations on $\Gamma_{d}$ is able to deblur the noisy data $\phi_{d}$. It also gives accurate reconstructions and is stable.

Secondly, we look at the algorithm stability when the data $\psi_{d}$ is noisy. Figure 11 gives the noisy data used by the algorithm. Figures 12 and 13 give the function and its normal derivative reconstruction.

We can notice that the first order method, by opposition to the previous one [6], is also able to deblur the noisy data $\psi_{d}$. The deblurring effect of the first order method does not rely on the use of the interpolation by B-Splines for generating the additional data. The justification comes from the following points:

- The zero order method (previous method) already deblurred the field data $\phi_{d}$ but not the flux data $\psi_{d}$,

- The first order method behaves roughly like three zero order methods for the evaluation of $u$ and its two partial derivatives $u_{1}$ and $u_{2}$. So it deblurs $\phi_{d}, \phi_{1 d}$ and $\phi_{2 d}$ data and therefore the flux data $\psi_{d}$.

\subsubsection{Non smooth boundary}

Numerical simulations are now performed on a square domain $(\Omega=] 0,1[\times] 0,1[)$. The $\Gamma_{d}$ boundary part is composed of two sides $(y=0$ and $x=1)$ (Figure 14). The boundary mesh is a regular one with 120 elements on each side. 


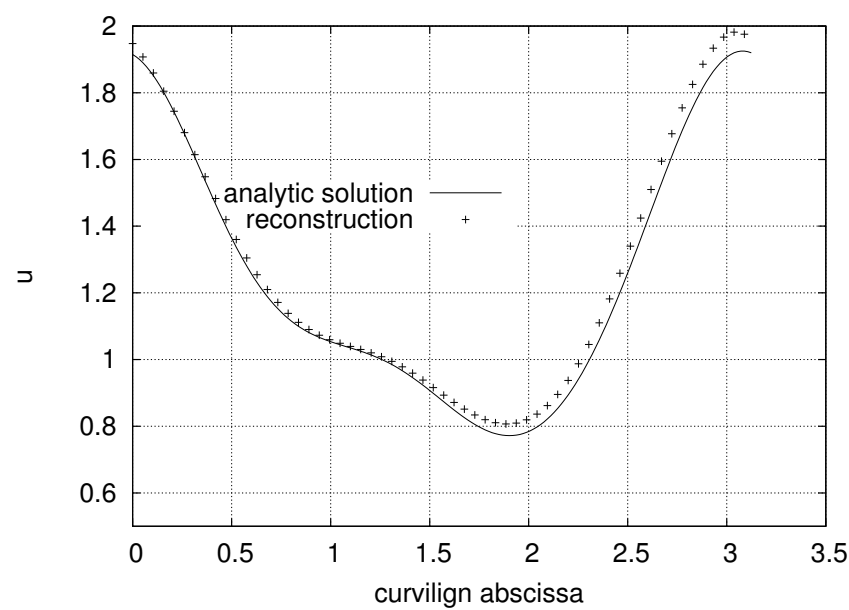

Fig. $9 u$ first order method reconstruction ( $\phi_{d}$ noisy data (10\%))

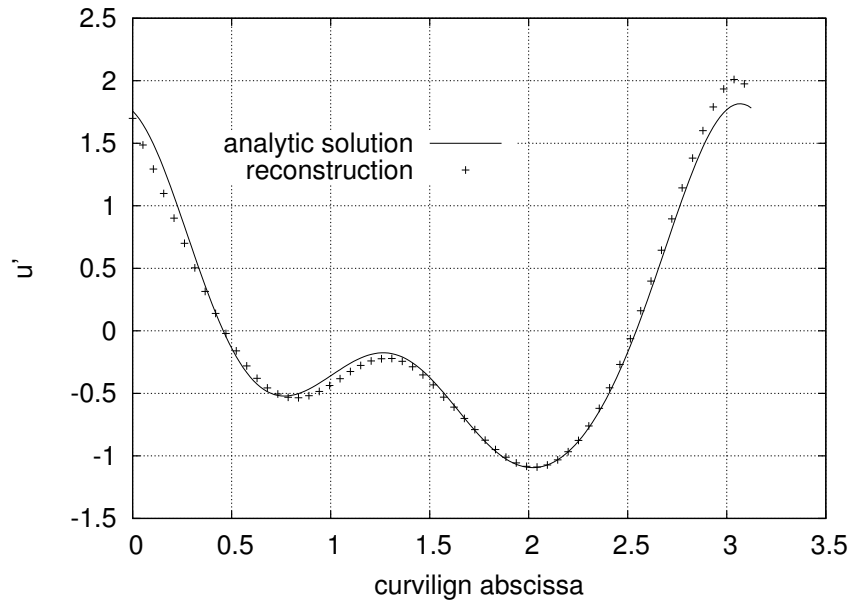

Fig. $10 u^{\prime}$ first order method reconstruction ( $\phi_{d}$ noisy data (10\%))

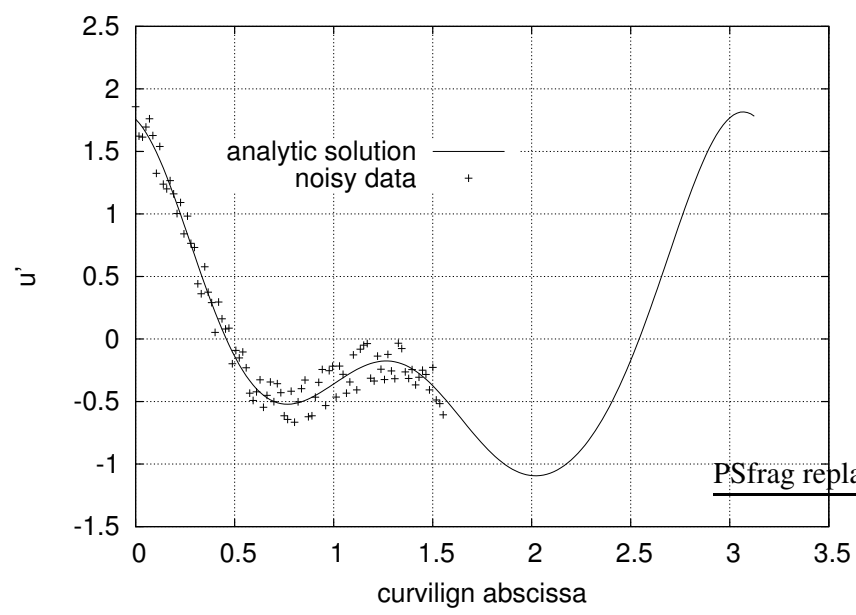

Fig. $11 \psi_{d}$ noisy data (10\%)

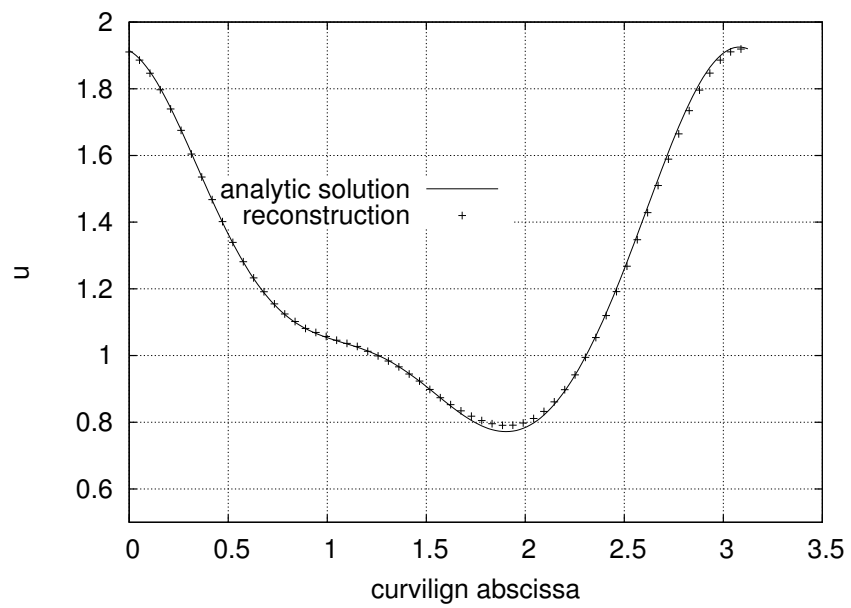

Fig. $12 u$ first order method reconstruction ( $\psi_{d}$ noisy data (10\%))

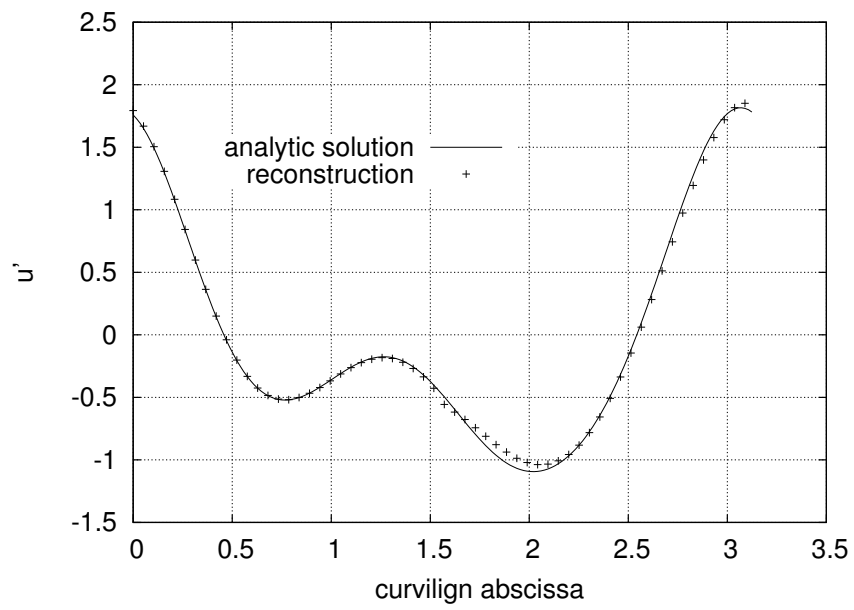

Fig. $13 u^{\prime}$ first order method reconstruction ( $\psi_{d}$ noisy data (10\%))

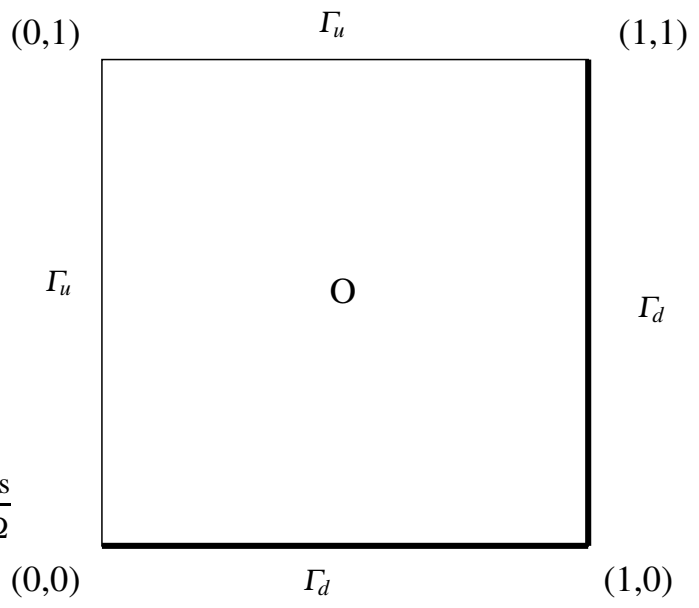

Fig. 14 Domain with non smooth boundary 


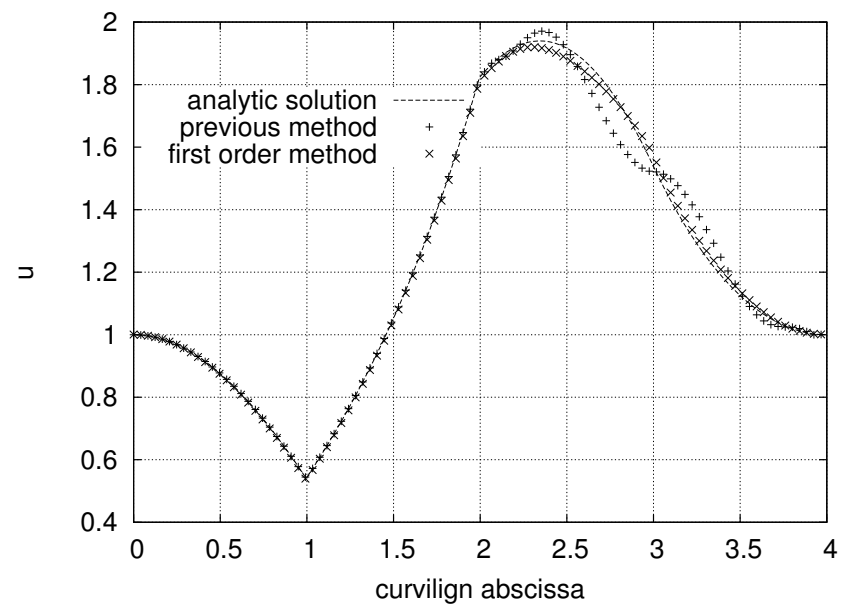

Fig. $15 u$ reconstructions (previous and first order method)

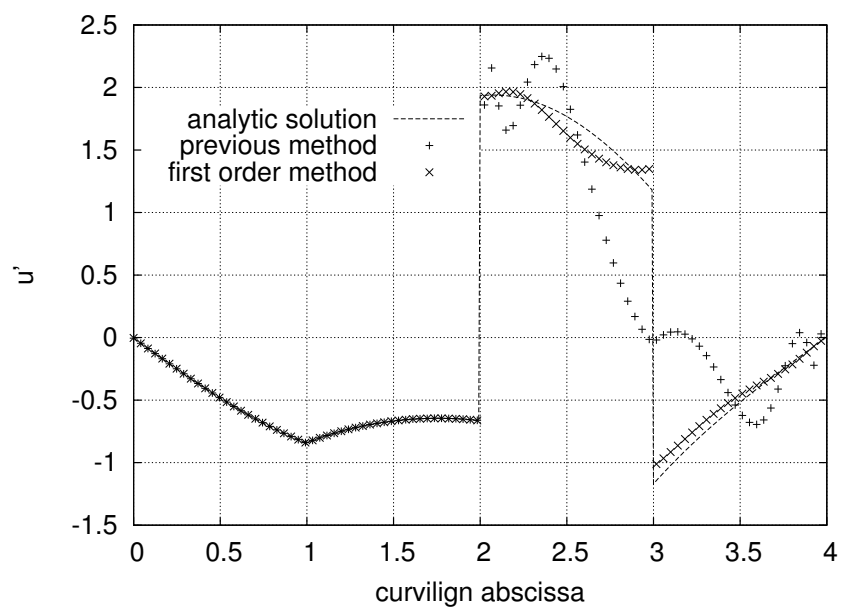

Fig. $16 u^{\prime}$ reconstructions (previous and first order method)

\section{Comparison with other methods}

This example was already studied by our previous method [6], a Tikhonov like method [11] and an iterative method [16] which all gave similar results. Figure 15 (respectively Figure 16) compares function (respectively normal derivatives) reconstructions obtained with one of these methods [6] and with the first order method. We can notice improved reconstructions by the first order method.

\section{Behaviour with respect to noisy data $\phi_{d}$ and $\psi_{d}$}

We look at the algorithm stability when the $\phi_{d}$ and $\psi_{d}$ data are both noisy. Figure 17 and Figure 18 give the function and its normal derivative reconstruction in this case and show the efficiency and the stability of this new approach. In particular, it is stable with respect to perturbations on the data $(1 \%$ noise level).

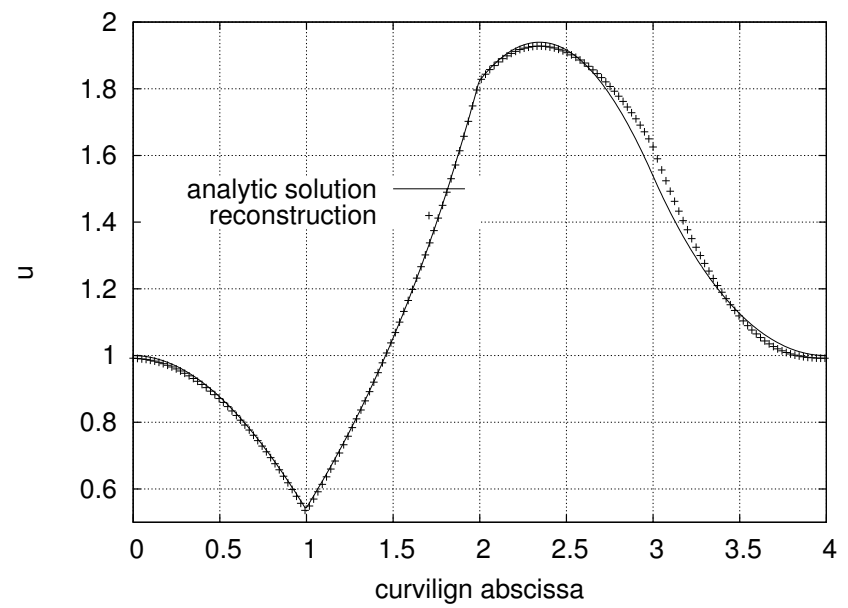

Fig. $17 u$ first method reconstruction (noisy data $\phi_{d}$ and $\psi_{d}(1 \%)$ )

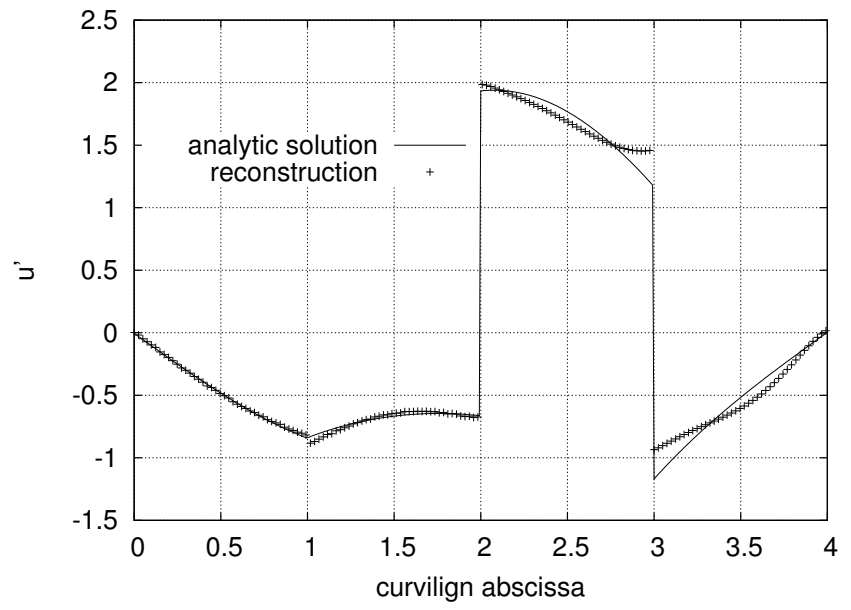

Fig. $18 u^{\prime}$ first method reconstruction (noisy data $\phi_{d}$ and $\psi_{d}(1 \%)$ )

\section{Conclusion}

A new method has been introduced for Cauchy inverse problems. This method has been carried out for a model problem. It has introduced a higher order problem which has been numerically implemented using BEM.

Although this new method requires a computational time approximately nine times greater than that of the previous method, the presented numerical simulations prove the efficiency, the accuracy and the robustness of the method as well as the following properties:

- It reconstructs the function like its normal derivative with an increased precision,

- It allows us to reduce the extension of the boundary part $\Gamma_{d}$ where prescribed data are given,

- It deblurs the two boundary data $\phi_{d}$ and $\psi_{d}$ when they are noisy.

This method can be extended without difficulty to 3D problems as well as to other problems, like elasticity problems. 
Acknowledgements The authors would like to thank reviewers for their relevant remarks and comments.

\section{References}

1. Andrieux S, Baranger T, Ben Abda A (2006) Solving Cauchy problems by minimizing an energy-like functional. Inverse Problems 22:115-133

2. Bonnet M (1999) Boundary Integral Equations Methods in Solids and Fluids. John Wiley and Sons, Chichester

3. Bourgeois L (2005) A mixed formulation of quasi-reversibility to solve the Cauchy problem for Laplace's equation. Inverse Problems 21:1087-1104

4. Cimetière A, Delvare F, Jaoua M, Pons F (2001) Solution of the Cauchy problem using iterated Tikhonov regularization. Inverse Problems 17(3):553-570

5. Cimetière A, Delvare F, Pons F (2005) Une methode inverse d'ordre un pour les problèmes de complétion de données. Comptes Rendus Mecanique 333(2):123-126

6. Delvare F, Cimetière A, Pons F (2002) An iterative boundary element method for Cauchy inverse problems. Computational Mechanics 28(3-4):291-302

7. Eilers P, Marx B (1996) Flexible smoothing with B-splines and penalties. Statistical Science 11(2):89-121

8. Engl H, Hanke M, Neubauer A (1996) Regularization of Inverse problems. Kluwer Academic Publishers, Dordrecht

9. Fasino D, Inglese G (1999) An inverse Robin problem for the Laplace's equation: Theorical results and numerical methods. Inverse Problems 15:41-48

10. Hadamard J (1953) Lectures on Cauchy's problem in Linear Partial Differential Equation. Dover, New-York

11. Hayashi K, Ohura Y, Onishi K (2002) Direct method of solution for general boundary value problem of the Laplace equation. Eng Anal Bound Elem 26:763-771

12. Jourhmane M, Lesnic D, Mera N (2004) Relaxation procedures for an iterative algorithm for solving the Cauchy problem for the Laplace equation. Eng Anal Bound Elem 28(6):655-665

13. Klibanov M, Santosa F (1991) A computational quasi-reversibility method for Cauchy problems for Laplace's equation. SIAM J Appl Math 51(6):1653-1675

14. Kozlov V, Maz'ya V, Fomin A (1991) An iterative method for solving the Cauchy problem for elliptic equations. Comput Math Phys 31(1):45-52

15. Lattès R, Lions JL (1967) Méthode de Quasi-réversibilité et applications. Dunod, Paris

16. Lesnic D, Elliott L, Ingham D (1997) An iterative boundary element method for solving the Cauchy problem for the Laplace equation. Eng Anal Bound Elem 20:123-133

17. Pasquetti R, Petit D (1995) Inverse diffusion by boundary elements. Eng Anal Bound Elem 15:99-107

18. Tikhonov A, Arsenin V (1977) Solutions of Ill-posed problems. John Wiley \& Sons, New-York 